\title{
Significados atribuidos por un grupo de hombres adultos a la experiencia de tener diabe- tes mellitus tipo 2
}

\author{
Meanings attributed by a group of adult men to the experience of having type 2 diabetes \\ mellitus
}

Gabriela Vanegas ${ }^{1}$, Alejandro Barbosa ${ }^{1 *}$ y Angie Zamora ${ }^{1}$

${ }^{1}$ Facultad de psicología, Universidad Antonio Nariño, Colombia.

\section{* Correspondencia: abarbosagonzalez@gmail.com.}

Recibido: 23 de enero de 2020 | Revisado: 01 de marzo de 2020 | Aceptado: 20 de abril de 2020 | Publicado Online: 01 de mayo de 2020

CITARLo COMo:
tipo 2. Interacciones, 6(2), e157. http://dx.doi.org/10.24016/2020.v6n2.157

\section{RESUMEN}

Introducción: La diabetes es una enfermedad crónica que causa diversas limitaciones en la vida personal, familiar y social de la persona que la padece. Objetivo: Se pretendía analizar el significado atribuido por un grupo de hombres adultos a la experiencia de tener diabetes mellitus tipo 2. Metodología: La metodología fue cualitativa con diseño narrativo. La técnica para recolectar la información una entrevista libre. El grupo de participantes estaba conformado por cuatro progenitores hombres adultos con diagnóstico de diabetes mellitus tipo 2. Para la selección se utilizó un muestreo intencional. Las entrevistas se grabaron en audio y se trascribieron literalmente. Se realizó un análisis categorial inductivo por medio de matrices. Allí se identificaron similitudes y diferencias entre participantes. Resultados: Dentro de los hallazgos se resalta que el impacto de la enfermedad se presenta en lo personal, pareja, familia y ámbito laboral. Las principales consecuencias físicas son problemas de visión, movilidad e insensibilidad en miembros inferiores. $A$ nivel psicológico sentimientos de tristeza. Conclusiones: La familia juega un papel fundamental a la hora de enfrentar la enfermedad, ya que se constituye en fuente de apoyo en la adherencia al tratamiento. Se evidenció en los hijos de los participantes una construcción de significados en torno a la enfermedad que minimiza el impacto de la misma. Lo anterior tiene el riesgo potencial para que se repitan comportamientos de riesgo en las generaciones posteriores.

Palabras clave: Significado, experiencia, diabetes mellitus tipo 2, hombres adultos.

\section{ABSTRACT}

Background: Diabetes is a chronic disease that causes various limitations in the personal, family and social life of the person suffering from it. Aim: It was intended to analyze the meaning attributed by a group of adult men to the experience of having diabetes mellitus type 2. The methodology was qualitative with narrative design. The technique to collect information was a free interview. The group of participants consisted of four adult male parents diagnosed with diabetes mellitus type 2 . Intentional sampling was used for the selection. The interviews were recorded in audio and literally transcribed. An inductive categorical analysis was performed by means of matrices. There, similarities and differences between participants were identified. Results: Among the findings it is highlighted that the impact of the disease is presented in the personal, couple, family and work environment. The main physical consequences are 
problems of vision, mobility and insensitivity in lower limbs. On a psychological level feeling of sadness. Conclusion: The family plays a fundamental role in dealing with the disease, since it constitutes a source of support in adherence to treatment. It was evident in the children of the participants a construction of meanings around the disease that minimizes its impact. The above has the potential risk for risk behaviors to be repeated in later generations.

Keywords: Meaning, experience, diabetes mellitus type 2, adult men.

\section{INTRODUCCIÓN}

La diabetes mellitus (DM) es considerada una enfermedad crónica no transmisible que se relaciona con altos niveles de glucosa debido al consumo de azucares y proteínas en exceso y la consecuente alteración de producción de insulina en el páncreas. Según el reporte de Diabetes Atlas (International Diabetes Federation) (IDF) (2019), a nivel mundial existen 463 millones de personas entre los 20 a 79 años diagnosticadas con diabetes con un potencial incremento del $51 \%$ hacia el año 2045. En América central y del sur, en el mismo rango de edad, existen 32 millones de personas con este diagnóstico que tiende a incrementarse en un 55\% para el año 2045. La prevalencia de la enfermedad en esta región y en este mismo rango de edad es de $9.4 \%$, siendo Brasil, Paraguay y Chile de los países con mayor porcentaje de adultos diagnosticados con diabetes. Adicionalmente la IDF (2019) estima que existen más de $25 \%$ de mujeres y $20 \%$ de hombres diagnosticados en la región de América Latina (América central y sur).

En Colombia se estima que 2 millones de personas han sido diagnosticadas; Sedó (2010) refiere que los hombres mayores suelen ser quienes reportan la mayor intensidad de los síntomas, ya que les genera sentimientos de inutilidad, inseguridad, aislamiento social y proximidad a la muerte. Debido a lo anterior, no generan conductas de adherencia al tratamiento (dieta alimenticia, ejercicio físico y farmacológico). Por otro lado, Pérez, Rivas y Martínez (2015), indican que algunos adultos no tienen problemas con el seguimiento del tratamiento y manejo de la diabetes, pero si les suscita malestar no consumir alimentos de su agrado.

También está el impacto psicológico. Para González, Tinoco y Benhumea (2011), algunas respuestas psicológicas significativas son el miedo, ansiedad, ira, depresión, sentimientos de culpa y temor de abandono por parte de la familia. A partir de esto podemos inferir que el apoyo familiar para el afrontamiento de la diabetes es fundamental. Al respecto Herrera, Quintero y Hernández (2007) argumentan que la red de apoyo le permite a la persona el acceso a la información y tratamientos. Para las personas con diabetes las relaciones familiares y sociales pueden cambiar ya que as enfermedades crónicas generan un impacto no solo en las personas que las padecen, sino también en su familia y red social. Dichos cambios pueden ser positivos (unión y apoyo en los momentos de dificultad), pero también negativos (aislamiento social e incomprensión). Dentro de la revisión del estado del arte hecha en el presente proyecto investigativo se observó la poca cantidad de estudios desde una perspectiva cualitativa con diseño narrativo que permita comprender las experiencias de personas diagnosticadas con diabetes. Por lo anterior, el objetivo de investigación consistía en analizar el significado atribuido por un grupo de hom- bres adultos a la experiencia de tener diabetes mellitus tipo 2 para generar un acercamiento a la experiencia de padecer esta enfermedad y ofrecer reflexiones en torno a su intervención.

\section{Diabetes Mellitus tipo 2}

Este tipo de diabetes se caracteriza por altos niveles de glucosa y es definido por la Organización mundial de la salud (OMS). (2018). como una enfermedad crónica que se origina cuando el cuerpo no asimila correctamente la insulina que se produce. La insulina es una hormona que regula el efecto del azúcar en la sangre y es por ello que alteraciones en su asimilación producen hiperglicemia que genera, a largo plazo, serios problemas de salud. Por ello, la persona diagnosticada debe seguir con rigurosidad el tratamiento médico que se enfoca en manejar la insulina para lograr el control de la glicemia. Según Gil, Sil, Domínguez, Torres y Medina (2013), es importante explicarle a la persona desde el momento del diagnóstico, que la insulina es necesaria dependiendo de la evolución de la enfermedad. Lo anterior es importante, ya que la educación terapéutica es clave para el manejo adecuado de la enfermedad.

\section{Familia y enfermedad}

La familia contemporánea es el producto de diversos cambios históricos y culturales. Actualmente existen diferentes tipos de conformaciones familiares. Como ejemplo tenemos la familia nuclear, recompuesta, uniparental y monoparental entre otras. En cada familia se presentan características particulares asociadas con el ciclo vital, comunicación, los roles y la jerarquía (Barbosa \& Jiménez, 2010). En general, la familia es parte del grupo de apoyo con el que cuentan las personas y que suele pasar por diferentes etapas en su proceso de evolución y cambio permanente. Todo lo anterior implica desafíos adaptativos para el grupo y cada uno de sus integrantes.

Uno de los estresores que puede afectar la armonía familiar es la aparición de una enfermedad crónica. Con relación al tema Rolland (2000) argumenta que la persona con una enfermedad crónica tiene miedo de que su vida termine antes que su proyecto de vida, ya que la aparición de una enfermedad interrumpe el proyecto de vida. $\mathrm{n}$ algunos casos los padres pasan a ser cuidados por sus propios hijos. Situación que puede generar conflictos familiares intergeneracionales (Ruiz, 2012; Placeres \& León, 2011). Los hombres adultos al percibir el impacto de la enfermedad en su vida cotidiana le otorgan un sentido particular (varia de persona a persona) que nace de un conglomerado de interacciones familiares, creencias socio culturales y particularidades individuales. Según Heredia y Pinto (2008), la persona diagnosticada con una enfermedad crónica necesita comprender y controlar los procesos relacionados con la vida cotidiana. 


\section{MÉTODO}

\section{Tipo de investigación y diseño}

La investigación se orienta desde el enfoque cualitativo. Lo anterior porque se pretende rastrear la realidad desde la perspectiva de los participantes. El interés de la investigación está centrado en el significado, la narración, la comprensión y la acción (Rodríguez, 2011).

En cuanto al diseño es narrativo, lo cual implica el uso de técnicas de recolección de datos como las entrevistas individuales y grupales. Allí las personas pueden relatar sus experiencias de manera espontánea. Para Barbosa, Reyes y Escobar (2009), desde el mismo momento en que las personas narran sus experiencias vitales, actualizan su historia y las experiencias retornan a manera de imágenes. También Fernández, Hernández y Baptista (2014), argumentan que los diseños narrativos tienen que ver con la sucesión de hechos, fenómenos y procesos. Allí se involucran pensamientos, sentimientos e interacciones. Lo anterior se suscita gracias a la narración directa de los protagonistas. Finalmente, Cardona y Alvarado (2015), agregan que desde esta perspectiva se comprende la realidad como una construcción social. Debido a lo anterior, la aproximación al mundo está mediada por los sujetos y su experiencia.

\section{Técnica para recabar la información}

La técnica utilizada fue la entrevista no estructurada, la cual busca la información que se necesita por medio de unos temas definidos o focos. Díaz, Torruco, Martinez y Varela (2013) plantean que este tipo de entrevista es informal y flexible, por el cual se adapta a las condiciones contextuales. Sin embargo, esto no impide llegar a la profundización del tema y detallar aspectos relevantes para la investigación. Por medio de la pregunta y respuesta se logra la reconstrucción de vida de la persona entrevistada y en su relato se ve reflejado el significado que la persona tiene sobre una vivencia o tema abordado, en este caso la experiencia de padecer Diabetes Mellitus tipo 2.

\section{Participantes}

Para esta investigación se seleccionó a cuatro participantes. Los criterios de inclusión consistían en: ser hombres adultos (50-70 años de edad), tener diagnóstico de diabetes mellitus tipo 2, ser padres y contar con por lo menos estudios secundarios; se seleccionó esta población ya que como lo refiere Sedó (2010), los adultos mayores pueden resentir con mayor intensidad los efectos de la diabetes; Adicionalmente a esto la diabetes melli- tus tipo 2 es más recurrente en adultos que en otro grupo etario. (Palacios-Barahona, Arango-Posada, Ordoñez, Alvis-Guzmán. 2019).

A continuación, se describen los datos demográficos de los participantes. Lo anterior teniendo en cuenta el principio de confidencialidad.

\section{Procedimiento}

En un inicio se ubicaron a 12 participantes potenciales pertenecientes a un proyecto investigativo de la Universidad Antonio Nariño sede Bogotá sobre prevención de la diabetes. Tras realizar una entrevista exploratoria se establecieron los criterios de inclusión y finalmente se seleccionaron los cuatro para las entrevistas abiertas. La técnica para la selección fue el muestreo intencional. Para la entrevista se realizaron dos encuentros. En el primero se explicitaron los objetivos y justificación del estudio. Después de generar empatía y resolver inquietudes sobre la participación en el trabajo, se concretó un segundo encuentro para la firma del consentimiento informado y la entrevista. La entrevista se grabó en audio para su posterior trascripción literal y análisis. El análisis desarrollado es del tipo categorial inductivo. Para tal fin se utilizaron matrices de sentido donde se organizaron los fragmentos de las entrevistas más recurrentes dentro de los relatos de los entrevistados. Con este material se infirieron las categorías que permitieron realizar el análisis. Dichas categorías fueron: Significados atribuidos al diagnóstico y aceptación de la enfermedad, significados atribuidos a las consecuencias psicológicas y adherencia al tratamiento; significados atribuidos a cambios en las relaciones con los hijos y la pareja. Finalmente, significados atribuidos a limitaciones en la vida sexual y laboral.

\section{RESULTADOS Y DISCUSIÓN}

\section{Significados atribuidos al diagnóstico y aceptación de la en-} fermedad

Esta categoría hace referencia a antecedentes médicos y ambientales de la enfermedad. También al impacto físico generado por la enfermedad. A partir de esto aparecen las experiencias asociadas con los síntomas y las limitaciones que esta enfermedad genera en las personas.

La mayoría de los participantes mencionan antecedentes diabéticos en su historia familiar. Sin embargo, no asocian este hecho con el desarrollo de esta enfermedad en su historia personal. Relato de Luis (3 años diagnosticado): Mis hermanos me contaron que mi abuelita falleció por diabetes. Santiago (17

Table 1. Datos demográficos de las participantes $(n=4)$

\begin{tabular}{lcccc}
\hline & Pedro & Alejandro & Luis & Santiago \\
\hline Edad & 65 años & 57 años & 57 años & 65 años \\
Nivel Educativo & Bachiller & Bachiller & Bachiller & Bachiller \\
Ocupación & Desempleado & Vendedor & Conductor & Conductor/ independiente \\
Tiempo de Diagnóstico & 20 años & 1 año y seis meses & 3 años & 17 años \\
Edad de los Hijos & $23,30,33$ y 35 & $34,32,26,23$ y 22 & 118 y 19 años & $21,22,28,37$ y 39 \\
Vive con & Madre & Esposa & Esposa y dos hijos & Hijas y sus nietos \\
\hline
\end{tabular}


años diagnosticado): Mi papá murió de diabetes y pues también mi hermano. Cuando me enteré que ellos tenían esa enfermedad, yo seguí todo tranquilo. Uno con buena salud, no piensa que se pueda enfermar de diabetes.

En cuanto a antecedentes ambientales, los participantes reconocen hábitos alimenticios que se caracterizan por la ingesta de comida poco saludable. Alejandro (1 año y seis meses diagnosticado): Pienso que me enferme por comer en la calle, comida chatarra. Fritos y gaseosas. Santiago (17 años diagnosticado): Prácticamente comía a deshoras, pero ahora tampoco puedo comer a horas fijas. Así mismo, Los antecedentes biológicos familiares, así como los hábitos alimenticios son reconocidos como elementos determinantes en el surgimiento de la enfermedad. Según Colombie, Socca, Rivas y Borrego (2016): “Antecedentes familiares de diabetes, hipertensión arterial (HTA), obesidad y factores ambientales juegan un papel importante en el surgimiento y desarrollo de la enfermedad" (p. 125). Para los participantes, los primeros síntomas de la enfermedad son los que encienden la alarma acerca de la situación, pero es a raíz del diagnóstico que se toma conciencia de que la diabetes es una enfermedad que coloca en riesgo la salud. Pedro (20 años diagnosticado): Yo estaba trabajando en Yopal, ese día fuimos a comer una picada, todo estuvo bien, pero luego me sentí mal. Estuve 20 días con mucha sed. No podía ir al baño y no me daban ganas de comer. Pensé que el almuerzo estaba descompuesto. Alejandro (1 año y seis meses diagnosticado): La diabetes es totalmente silenciosa, esa enfermedad no se manifiesta con nada. Un día se despierta. Uno siente mareo, nunca había sentido ese mareo. Me sentía totalmente mareado y también mucha sed, no sabía el por qué, entonces me fueron a hospitalizar. Con respecto al tema Sedó (2010) explica que la diabetes suele ser asintomática. Se pueden tener glucemias altas sin presentar síntomas. Los relatos de los participantes evidencian el desconocimiento generalizado de la DM2 en cuanto a características, factores predisponentes y consecuencias. La consecuencia de ello es que no actúan preventivamente a pesar de los antecedentes de la enfermedad en la familia. De la misma manera el diagnóstico de la enfermedad los toma por sorpresa. Alejandro (1 año y 6 meses diagnosticado): Fue sorpresivo, yo y mi esposa nos sorprendimos. A ella le habían dicho cuando yo tenía el cáncer que era pre diabética y pues ella se cuidó bastante por dos o tres meses, pero se curó. Y cuando me dicen que yo ya tengo diabetes, no sabíamos qué hacer. Luis (3 años diagnosticado): Cuando me enteré me asusté mucho, yo le conté a mi señora, también se asustó mucho y no teníamos conocimiento de la enfermedad. En cuanto a las limitaciones que genera la enfermedad, los participantes las reconocen y aceptan; Dichas limitaciones están asociadas a problemas de movilidad, disminución de la visión e insensibilidad en los miembros inferiores. Luis (3 años diagnosticado): Anteriormente corría y tenía fuerza, ahora no. He sentido deterioro en la visión y en algunos momentos me da lo del pie diabético. Pedro (20 años diagnosticado): Ahora no puedo caminar mucho o quedarme de pie. A veces estoy caminando y de repente me caigo con mi bastón. Además, he tenido varias complicaciones en la piel porque no tengo esa sensibilidad de antes. En ocasiones no siento los pies.

\section{Significados atribuidos a las consecuencias psicológicas y ad- herencia al tratamiento}

En esta categoría aparecen experiencias relacionadas con el malestar psicológico y también las dificultades de los participantes para lograr adherirse al tratamiento de forma adecuada. También se resalta el apoyo familiar y las creencias religiosas. Las consecuencias psicológicas se las asocian con mayor recurrencia a sentimientos de tristeza y soledad: Alejandro (1 año y 6 meses diagnosticado): Las psicológicas, pensar en lo que pasa en mi vida y tener miedo a morirme. Santiago (17 años diagnosticado): Si, me da mucha tristeza, me decaigo, pero me trato de controlar. Según diferentes autores (Rolland, 2000; Jiménez \& Dávila, 2007), las reacciones psicológicas de tristeza, temor a la muerte, soledad e incluso depresión, suelen ser comunes en individuos con enfermedades crónicas; a esto se le puede sumar que el tratamiento de la diabetes requiere de un cambio significativo de las conductas de vida lo que ocasiona reacciones emocionales de angustia y frustración. (Canales \& Barras. 2014).

La principal fuente de información sobre la DM2 son los médicos dietistas, quienes realizan orientaciones indispensables para obtener mayor adherencia al tratamiento. Los participantes mencionan seguir las recomendaciones médicas como toma y aplicación de los medicamentos, cuidado nutricional y actividad física. Paradójicamente evidencian dificultades para seguir las mismas indicaciones. Esto debido a que algunas personas se auto medican o manejan creencias negativas con respecto a los medicamentos prescritos por el médico. Pedro (20 años diagnosticado): Me levanto y hago el desayuno, después medias nueves, almuerzo, tomo onces, como y a las 10:00 me coloco la insulina, me aplico $10 \mathrm{~cm}$ de insulina en el estómago y mucho cuidado alimenticio. Luis (3 años diagnosticado): Pues los médicos me dijeron que me cuidara en mis alimentos. Desde alli he venido juicioso. Por eso me tomo un cafecito con pan integral, pero en ocasiones pienso que prefiero morirme llenito antes que con hambre. Santiago (17 años diagnosticado): Yo no quería la insulina y así dure por tres meses, me aplicaba insulina y la mezclaba hierbas. Yo mismo me la suspendí, pero seguía tomándome cuatro pastillas. En relación con el cumplimiento y dificultades con la adherencia al tratamiento en los progenitores, Alves, Castro, Ribeiro y Faustino (2007) refieren que para que exista una evolución favorable en el tratamiento de la diabetes debe existir una autorregulación de la conducta. Lo anterior requiere un compromiso y esfuerzo que faciliten la adherencia al tratamiento.

Hoyos, Arteaga \& Muñoz (2011), identifican, en su investigación acerca de factores que limitan el tratamiento en pacientes diabéticos, las percepciones emocionales, alimentarias y religiosas como punto de inflexión en el tratamiento de diabéticos; al mismo tiempo proponen la comprensión de creencias socioculturales como parte del proceso interventivo para aumentar la eficacia del tratamiento. Sin embargo y a pesar de todo lo anterior, los participantes del estudio se muestran motivados para continuar luchando contra la enfermedad. Lo que descrito a continuación tiene que ver más con su familia que con sus proyectos de vida o ellos mismos. Luis (3 años diagnos- 
ticado): Me tomo mis medicamentos por mis hijos y mi señora, mi familia. Quiero estar bien para ellos.

\section{Significados atribuidos a cambios en las relaciones con los hi-} jos y la pareja

En este caso se evidencian experiencias asociadas a cambios en las interacciones familiares que tienen que ver con la aparición de la enfermedad. Dentro de dichos cambios se encuentra la reacción de apoyo de los hijos de los participantes. Igualmente, se observa el papel de la pareja en torno a la adherencia al tratamiento y al asumir responsabilidades jerárquicas.

En las narraciones de los participantes predomina el tema de la individuación de los hijos y la conformación de nuevos hogares. Lo cual repercute en la construcción de una nueva dinámica familiar, Pedro (20 años diagnosticado): No digo que mis hijos no me colaboran, pero si siento como si estuviera pidiendo limosna y las veces que me he enfermado, le cuento a mi hija. Ella está a mi lado. Mis hijos tienen que responder por su hogar, ella quiere estar conmigo, pero no puede. Luis ( 3 años diagnosticado): Desde la enfermedad nosotros seguimos compartiendo igual, no compartimos la misma comida, pero las otras cosas sí. Mis hijos llaman y están pendientes de mí. Aunque ellos ya tienen sus hogares. Santiago (17 años diagnosticado): Me he alejado mucho de mis hijas menores, ellas ya están haciendo su vida. Los hijos de los participantes se alejan debido a sus responsabilidades y este alejamiento posiblemente conlleve una desinformación en torno a una enfermedad que es potencialmente heredable. Alejandro ( 1 año y 6 meses diagnosticado): Siento que para ellos todo lo relacionado con la enfermedad es normal, quizás porque piensan que el cáncer era más duro que la diabetes, y como salí del cáncer, pues creen que puedo salir de esta enfermedad, que no necesito su apoyo. Se evidencia en los hijos de los participantes una construcción de significados en torno a la enfermedad que minimiza el impacto de la misma. Lo anterior puede influir para que se repitan comportamientos de riesgo en las generaciones posteriores. En este caso se puede heredar la enfermedad y también las creencias y comportamientos asociados al problema. Para Rolland (2000) lo asintomático de una enfermedad crónica refuerza la confusión y desorientación de diversas formas. Esto facilita el uso de la negación en las personas con la enfermedad y sus familiares. El resultado es la minimización de los efectos de la enfermedad. También la pareja de los participantes constituye una importante fuente de apoyo para el manejo del tratamiento. Alejandro (1 año y 6 meses diagnosticado): Ha cambiado bastante, se sale de lo normal. La vida en pareja cambió. Ahora nosotros hablamos mucho de nuestras enfermedades y pues también de la empresa, pero más de la enfermedad. Ella siempre ha estado conmigo y más en mis enfermedades. Luis (3 años diagnosticado): Al inicio de la enfermedad todo empezó a cambiar. Yo he tratado de controlar mi enfermedad para compartir con ella. He aprendido a vivir con la enfermedad, creo que todos hacemos eso y más ella porque es quien está conmigo. En ocasiones ella se tiene que hacer responsable de imponer normas y asumir ciertas responsabilidades que antes me correspondían. Debido a las limitaciones generadas por la enfermedad, aparecen cambios en cuanto a la distribución de las responsabilidades y manejo de la autoridad. En la mayor parte de casos, es la pareja de los participantes quien asume responsabilidades y autoridad para equiparar la jerarquía familiar. Según Mendizábal y Pinto (2006), es importante que dentro de las interacciones familiares exista una jerarquía clara. Esto independientemente del momento vital y de la situación enfrentada. Todo lo anterior se relaciona con lo expresado por Hernández (1997), quien señala que el verdadero reto familiar está en enfrentar estresores no normativos, como es el caso de la enfermedad crónica, reorganizándose como una forma de recurso familiar que en últimas le permite a la familia adaptarse a nuevas situaciones.

\section{Significados atribuidos a limitaciones en la vida sexual y lab- oral}

En esta categoría se condensan las limitaciones más significativas que los participantes expresan en la experiencia de tener DM2. Particularmente en la vida sexual y laboral. Se destacan experiencias asociadas a dificultades físicas y psicológicas

La vida sexual en la pareja disminuye significativamente. Esto se ve ligado a las consecuencias físicas de la enfermedad. Allí se observan alteraciones en el control del cuerpo y consecuencias psicológicas como la depresión que disminuye el deseo hacia la otra persona. Pedro (20 años diagnosticado): Uno lo pierde todo, por ejemplo, ya uno no le da las ganas de estar con ella. Eso es instantáneo y la pila no funciona. Lo primero que piensan ellas es que uno tiene a otra persona. Luis (3 años diagnosticado): Existen momentos en que todo cambia con la pareja. Yo antes era normal con mi pareja. Antes teníamos relaciones tres veces al mes y ahora una vez y eso. Alejandro (1 año y 6 meses): La vida en pareja se sale de lo normal, es un cambio muy drástico. Ya no hay de eso entre nosotros. Para Lendon y Castelo (2013), esta enfermedad produce deficiente control metabólico, síntomas depresivos, limitaciones sociales y vida sexual menos satisfactoria entre otras complicaciones que afectan la calidad de vida.

También se evidencia deterioro en el ámbito laboral, ya que el rendimiento no es el mismo. Lo anterior por las limitaciones físicas y el sentimiento de aislamiento social. Alejandro (1 año y 6 meses): Mientras yo trabaje puedo seguir con mi vida normal. El comercio me exige que debo seguir y cada día me pide más productos. Esto hace ampliar mi micro empresa y me deja más ganancias. Pero, me toca pedirle alguien también que me ayude en el trabajo. Es una limitación, no me puedo esforzar. Luis: La diabetes genera sueño, trato de dormir por ratos. Santiago (17 años diagnosticado): Al inicio sí, tuve algo más de dos años que no podía trabajar. Ahí se decayó todo, automáticamente me quedé en cero. Pero continué.

\section{CONSIDERACIONES FINALES}

Se observa dentro de los relatos de los participantes un desconocimiento de la diabetes y la falta de reconocimiento de los factores de riesgo que hacen posible su desarrollo. Esto a pesar de tener antecedentes familiares diabéticos. Como principales consecuencias físicas los participantes identificaron problemas de visión, movilidad e insensibilidad en miembros inferiores. A nivel psicológico sentimientos de tristeza y temor hacia el desarrollo de la enfermedad. 
Por otro lado, la familia juega un papel fundamental a la hora de enfrentar la enfermedad, ya que constituye una fuente de apoyo para la adherencia al tratamiento. La pareja de los participantes pasa a tener la mayor carga jerárquica y a veces económica. Se evidencia en los hijos de los participantes una construcción de significados en torno a la enfermedad que minimiza el impacto de la misma. Lo anterior tiene el riesgo potencial para que se repitan comportamientos de riesgo en las generaciones posteriores.

Además, la vida sexual en la pareja disminuye significativamente. Esto se ve ligado a las consecuencias físicas de la enfermedad. Allí se observan alteraciones en el control del cuerpo y consecuencias psicológicas como la depresión que disminuye el deseo hacia la otra persona. También se evidencia deterioro en el ámbito laboral, ya que el rendimiento no es el mismo. Lo anterior por las limitaciones físicas y el sentimiento de aislamiento social.

En cuanto a las limitaciones del estudio, el tiempo establecido para el desarrollo del proyecto no permitió ubicar a más participantes. Igualmente, no se contó con participantes mujeres para poder contrastar posibles diferencias y similitudes por género y rol dentro de las relaciones familiares.

\section{CONFLICTO DE INTERÉS}

Los autores expresan que no hubo conflictos de intereses al redactar manuscrito

\section{FINANCIAMIENTO}

El presente estudio fue autofinanciado

\section{ORCID}

Gabriela Vanegas https://orcid.org/0000-0003-1462-7720

Alejandro Barbosa http://orcid.org/0000-0003-1400-2042

Angie Zamora https://orcid.org/0000-0002-9629-2591

\section{REFERENCIAS}

Alves, J., Castro, C., Ribeiro, H. \& Faustino, H. (2007). La incertidumbre en la enfermedad y la motivación para el tratamiento en diabéticos tipo 2. Revista Latino Enfermagem, 15(4), 1-10. Recuperado de http://www.scielo.br/pdf/ rlae/v15n4/es_v15n4a09.pdf

Atlas de Diabetes (International Diabetes Federation) (IDF) (2019). IDF Diabetes atlas. Novena edición.

Barbosa, A. \& Jiménez P. (2010). Significados asignados al establecimiento de vínculos afectivos y la posible conformación familiar por parte de jóvenes adultos homosexuales. Revista Vanguardia Psicológica, 1(1), 33-51.

Barbosa, A., Reyes, S. \& Escobar, M. (2009). Narrativas dominantes de personas privadas de la libertad en torno al concepto de familia. Umbral Científico, (14), 170-180.

Canales \& Barra, E. (2014). Autoeficacia, apoyo social y adherencia al tratamiento en adultos con diabetes mellitus tipo II. Psicología y salud. Vol 24 Num. 2: 167-173. Recuperado de: http://psicologiaysalud.uv.mx/index.php/ psicysalud/article/view/922

Cardona, A. \& Alvarado, S. (2015). Investigación narrativa: apuesta metodológica para la construcción social de conocimientos científicos. Revista Ces psicología, 8(2), 171-181. Recuperado de http://revistas.ces.edu.co/index. $\mathrm{php} / \mathrm{psicologia/article/view/3022/2427}$

Columbíe, Y., Soca, P., Rivas, D. \& Borrego, V. (2016). Factores de riesgo asociados con la aparición de diabetes mellitus tipo 2 en personas adultas. Revista Cubana de endocrinología 27(2), 123-133. Recuperado de http://scielo.sld.cu/ pdf/end/v27n2/end02216.pdf

Díaz, L., Torruco, U., Martinez, M. \& Varela, M. (2013). La entrevista, recurso flexible y dinámico. Revista investigación en Educación Médica, 2(7), 162-167. Recuperado de https://www.redalyc.org/pdf/3497/349733228009.pdf
Fernández, R. Hernández, C. \& Baptista, M. (2014). Metodología De La Investigación. Recuperado de http://observatorio.epacartagena.gov.co/wpcontent/uploads/2017/08/metodologia-de-la-investigacion-sexta-edicion. compressed.pdf

Gil, L., Sil, M., Domínguez, E., Torres, P. \& Medina, J. (2013). Guía de práctica clínica diagnóstico y tratamiento de la diabetes mellitus tipo 2. Revista Med inst seguro soc, 51(1) 104-119. Recuperado de https://www.medigraphic. com/pdfs/imss/im-2013/im1310.pdf

González, N., Tinoco, A. \& Benhumea, L. (2011). Salud mental y emociones en pacientes con enfermedades crónico- degenerativas. Un acercamiento a la diabetes mellitus tipo 2. Revista Espacios públicos, 14(2), 258-279. Recuperado de http://www.redalyc.org/pdf/676/67621319013.pdf

Heredia, J. \& Pintó, B. (2008). Depresión en diabéticos: un enfoque sistémico. Revista Ajayu, 6(1), 22-41. Recuperado de http://www.scielo.org.bo/pdf/rap/ v6n1/v6n1a2.pdf

Hernández, A. (2009). Familia, ciclo vital y psicoterapia sistémica breve. Bogotá: Búho.

Herrera, L., Quintero, O. \& Hernández, (2007). Funcionalidad y red de apoyo familiar en pacientes diabéticos tipo 2 servicio de endocrinología lahula Mérida. Revista Academia, 6(12), 62-72. Recuperado de http:// www.saber.ula.ve/bitstream/handle/123456789/27301/articulo6. pdf;isessionid=04E4AD2DE1024D0956270EA7BCDAA4BF? sequence=1

Hoyos, T., Arteaga, M \&Muñoz, M. Factores de no adherencia al tratamiento en personas con diabetes Mellitus tipo 2 en el domicilio. La visión de un cuidador. Invec. Educ. Enferm. 29(2): 194-203. Recuperado de: http://www.scielo.org. co/scielo.php?script=sci abstract\&pid=S0120-53072011000200004

Jiménez, C. \& Dávila, M. (2007). Psicodiabetes. Revista Avances en psicología Latinoamericana, 25(1), 126-143. Recuperado de http://www.scielo.org.co/ scielo.php?script=sci arttext\&pid=S1794-47242007000100012

Lendon, L. \& Castelo, E. (2013). Afrontamiento al distres relacionado con la disfunción eréctil en varones con diabetes mellitus tipo 2. Revista Cubana endocrinología, 24 (3), 242 -257. Revisado de http://scielo.sld.cu/pdf/end/ v24n3/end02313.pdf

Mendizabal, O. \& Pinto, B. (2006). Estructura y diabetes. Revista Ajayu, 4(2), 191-211. Recuperado de http://www.scielo.org.bo/pdf/rap/v4n2/v4n2a3.pdf Organización Mundial de la Salud (OMS). (2018). Recuperado de: https://www. who.int/es/news-room/fact-sheets/detail/diabetes

Palacios-Barahona, U., Arango-Posada, M., Ordoñez, JE \& Alvis-Guzman, N. (2019). Calidad de vida de pacientes con diabetes tipo 2 en Colombia. Revisión sistemática y metaanálisis acumulativo. Rev. CES Psico. 12(3), 8090. Recuperado de: http://www.scielo.org.co/pdf/cesp/v12n3/2011-3080cesp-12-03-80.pdf

Pérez, Z., Rivas, V. \& Martínez, A. (2015). Percepción del apoyo familiar y dificultades relacionadas con la diabetes en el adulto mayor. Revista Horizonte sanitario, 14(1), 14-20. Recuperado de https://biblat.unam.mx/es/revista/ horizonte-sanitario/articulo/percepcion-del-apoyo-familiar-y-dificultadesrelacionadas-con-la-diabetes-en-el-adulto-mayor

Placeres, J. \& León, L. (2011). La familia y el adulto mayor. Revista Med. Electrón, 33(4), 472 - 483. Recuperado de http://scielo.sld.cu/pdf/rme/v33n4/ spu10411.pdf

Rodríguez, J. (2011). Métodos de investigación cualitativa. Revista de la corporación Internacional para el Desarrollo Educativo, (8), 1-34. Recuperado de http://www.cide.edu.co/doc/investigacion/3.\%20metodos\%20de\%20 investigacion.pdf

Rolland, J. (2000). Familias, enfermedad y discapacidad. Una propuesta de la terapia sistémica. España, Barcelona. Gedisa.

Ruiz, J. (2012). Aspectos biopsicosociales que incides en la salud del adulto. Revista Cubana de medicina general integral, 28(2), 79-86. Recuperado de http://scielo.sld.cu/pdf/mgi/v28n2/mgi09212.pdf

Sedó, P. (2010). Percepciones sobre la diabetes y su padecimiento en personas adultas mayores diabéticas y familiares en un área urbana de Costa Rica. Revista Anales en Gerontología, 6(6), 99-112. Recuperado de https://dialnet. unirioja.es/servlet/articulo?codigo $=6140284$ 